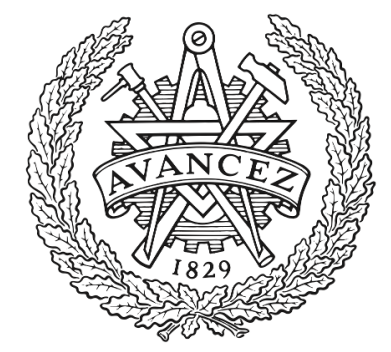

CHALMERS

UNIVERSITY OF TECHNOLOGY

\title{
Steering Redundancy for Self-Driving Vehicles using Differential Braking
}

Downloaded from: https://research.chalmers.se, 2023-04-26 01:36 UTC

Citation for the original published paper (version of record):

Jonasson, M., Thor, M. (2018). Steering Redundancy for Self-Driving Vehicles using Differential Braking. Vehicle System Dynamics, 56(5): 791-809.

http://dx.doi.org/10.1080/00423114.2017.1356929

N.B. When citing this work, cite the original published paper. 


\title{
Steering Redundancy for Self-Driving Vehicles using Differential Braking
}

\author{
M. Jonasson ${ }^{\mathrm{a}, \mathrm{b} *}$ and M. Thor ${ }^{\mathrm{a}}$ \\ ${ }^{a}$ Volvo Cars, Active Safety and Vehicle Dynamics Functions, Gothenburg, SE-405 31, Sweden; \\ ${ }^{b}$ The Royal Institute of Technology (KTH), Vehicle Dynamics, Department of Aeronautical and \\ Vehicle Engineering, Stockholm, SE-10044, Sweden
}

\begin{abstract}
This paper describes how differential braking can be used to turn a vehicle in the context of providing fail-operational control for self-driving vehicles. Two vehicle models are developed with differential input. The models are used to explain the bounds of curvature that differential braking provides and they are then validated with measurements in a test vehicle. Particular focus is paid on wheel suspension effects that significantly influence the obtained curvature. The vehicle behavior and its limitations due to wheel suspension effects are, owing to the vehicle models, defined and explained. Finally, a model based controller is developed to control the vehicle curvature during a fault by differential braking. The controller is designed to compensate for wheel angle disturbance that is likely to occur during the control event.
\end{abstract}

Keywords: differential braking; vehicle control; autonomous vehicles; redundant steering; scrub radius

\section{Introduction}

One of the goals of safety-critical systems is that a fault should not result in system failures. Since a self-driving vehicle isn't driven by a human driver, fail-operational ability must be inherent in the control design of the vehicle. Potential risks, addressed in this paper, are faults leading to failures with no steering capacity due to for example steering actuator faults, power black out, communication shut down etc. Those risks could, if the design is not fail-operational, lead to a failure and a hazard threatening the passengers safety. During, and directly after, a fault occurs, the vehicle should be able to be controlled into a safe state which is often terminated by a full stop.

The work in this paper is a result from further development of another paper [1] presented at Proceedings of the 13th International Symposium on Advanced Vehicle Control. Both papers contain an analysis of the temporary use of differential braking to regain control of the vehicle in case of faults. Differential braking used for cornering is described in e.g. [2,3]. Few descriptions are however found in the literature of how wheel suspension geometry influences the curvature response during differential braking and analysis from experiments in real world vehicles are rare. In [1], a simple vehicle model was presented and used. The simple vehicle model has in this paper been extended to also include the steering system model with wheel suspension parameters such as scrub

\footnotetext{
*Corresponding author. Email: mats.jonasson@volvocars.com
} 
radius. Particular focus has here been made on modelling the steering friction. The model validation shows that the new extended model has, compared with the simple one, a better match to the handling measurements. Owing to the developed vehicle model in this paper, it has also been possible to analytically express system constraints and maximum curvature as a function of wheel suspension parameters. This is important knowledge when designing the vehicle to secure a desired cornering ability.

The outline is as follows; Section 2 describes the underlying problem that seeks a solution by differential braking and Section 3 describes the vehicle model that will be used throughout the paper. The model is validated by making comparisons of measurement data from a test vehicle. In Section 4 the vehicle model is used to calculate the maximum curvature that is possible to obtain during differential braking at steady-state. The differential braking generates longitudinal tyre forces, and together with the wheel suspension geometry, a steering alignment torque is induced. Section 5 discusses the scrub radius geometry and how it contributes the resulting curvature. Two different alignments of scrub-radius is experimentally tested by experiments and evaluated. To explain the scrub radius effect of curvature and steering angle, a vehicle model including steering dynamics and scrub radius is developed in Section 6. In Section 7 a differential braking curvature controller is developed. The controller is developed to compensate for front wheel angle disturbance induced by the differential braking.

\section{VEHICLE AND PROBLEM DESCRIPTION}

The vehicle in this work is a conventional passenger car with front axle drive and steering. The mechanical steering system is provided with a steering actuator, mentioned to as Electric Power Assisted Steering (EPAS), which overlay an additive steering torque. Moreover, the vehicle has a friction brake system where brake torque can be applied at each wheel. See Table 1 for vehicle data.

In general the vehicle controllability during a steering failure depends on:

(1) Character of steering failure

(2) Initial condition of vehicle

(3) Road boundaries ahead

(4) Character of vehicle

(5) Character of failure detection and control algorithms

(6) Disturbances during the control

The character of the steering failure is typically described as too little or much steering torque. The initial condition of the vehicle are states related to position and orientation with respect to the road, state of the car itself including the actuators. Road boundaries ahead are the road curvature, road width and obstacles. The failure detection is typically characterized by the time to detect the failure and the correctness of the detection. Disturbances here means eg. rutted road, wind gusts etc.

One special case of a steering failure, among the many dependencies mentioned above, is when the torque from the steering actuator completely disappears. At the same time, the driver is assumed to not take part in controlling the vehicle and is not applying any steering-wheel torque. Figure 1 illustrates the obvious road departure that occurs when the steering does not provide torque when entering a curve from straight ahead initial driving. After approximately $20 \mathrm{~m}$ the vehicle is outside a 1 meter pre-defined lateral road margin. A second special case, as illustrated in Figure 2, is when the steering torque disappears in a curve i.e. the vehicle has cornering as initial motion state. To 
simulate the vehicle dynamic behavior after such a failure, an real world experiment has been done where the steering torque was forced to zero in a curve at $70 \mathrm{~km} / \mathrm{h}$ at $t=0$ and approx. $X=0$. As illustrated in Figure 2, the test vehicle will quickly leave the orbital motion and departure the road. When the steering torque from EPAS disappears, the alignment torque generated at the front axle will induce a change of the steeringwheel angle (SWA) towards zero within approximately $0.8 \mathrm{~s}$. This special case is in the context of self-driving vehicles most likely an unusual case due to the initial high lateral acceleration (approximately $7 \mathrm{~m} / \mathrm{s}^{2}$ ) which is outside any conceivable comfort zone. The case is however selected to demonstrate how quickly the vehicle leaves the road if no control is performed to reduce the effect of the failure.

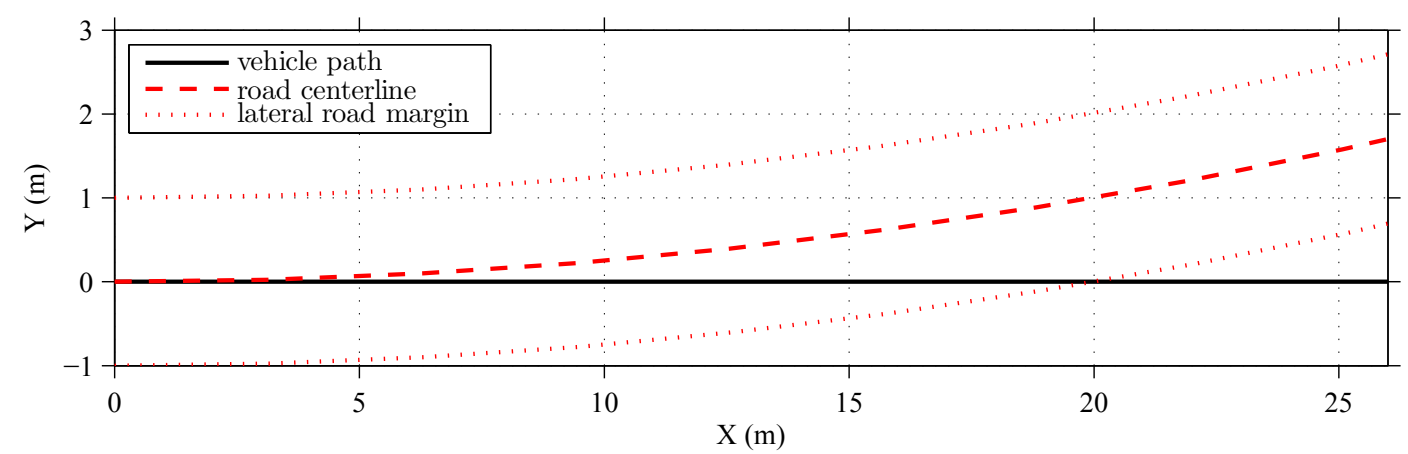

Figure 1. Effect of vanishing steering torque before entering a curve with $200 \mathrm{~m}$ radius and initially driving straight ahead (from simulation).
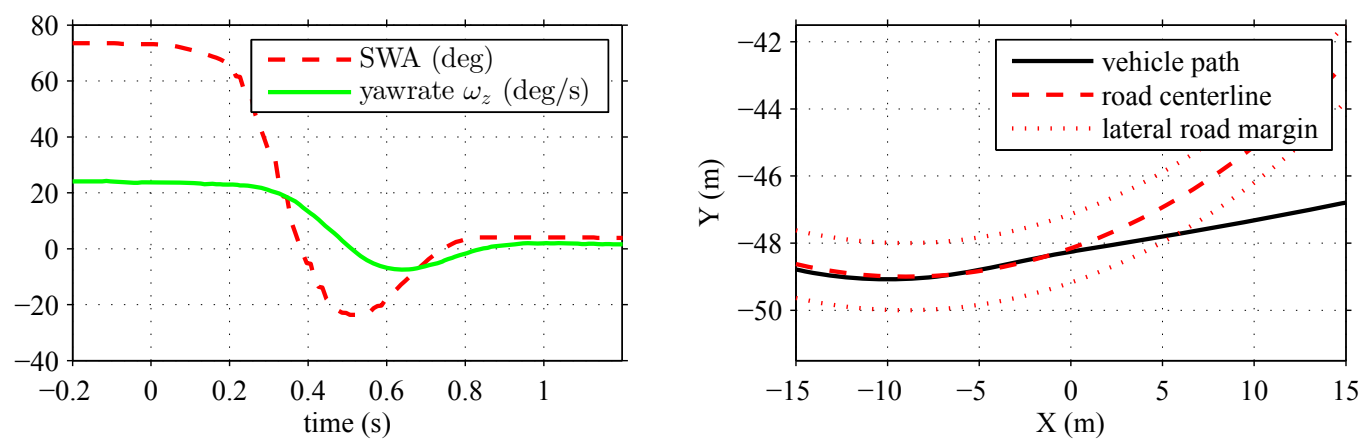

Figure 2. Effect of vanishing steering torque in a curve with $50 \mathrm{~m}$ radius and $70 \mathrm{~km} / \mathrm{h}$ (from vehicle measurements).

To cope with the problems described above, i.e follow the desired path without lateral tracking error after a steering capacity failure, the work in this paper addresses the use of differential braking to control the path. The proposed control strategy is intended to handle all dependencies mentioned above, but the work and the analysis performed are limited to investigate a complete loss of steering torque from EPAS and driving straight ahead initial condition.

The approach in this paper is to apply braking along one side of the vehicle to control the curvature. Since differential braking will influence the total longitudinal tractive force, they are coupled. It is for example not possible to maximize yaw torque and total brake force at the same time. The vehicle speed control is not part of the paper. 


\section{VEHICLE MODEL}

\subsection{Vehicle model description}

The well-established bicycle model with front steering input and constant vehicle speed assumption is here complemented with longitudinal tyre forces. See for example $[2,4]$ for a similar derivation. The longitudinal tyre forces are assumed to be small and limited to not influence lateral axle forces through combined slip. The model has lateral velocity $v_{y}$ and yawrate $\omega_{z}$ as states and is described by the lateral force and yaw torque equilibrium equations;

$$
\begin{aligned}
& m\left(\dot{v}_{y}+v_{x} \omega_{z}\right)=-\left(f_{x}^{F L}+f_{x}^{F R}\right) \sin \left(\delta_{f}\right)+\left(f_{y}^{F L}+f_{y}^{F R}\right) \cos \left(\delta_{f}\right)+f_{y}^{R L}+f_{y}^{R R} \\
& J_{z} \dot{\omega}_{z}=\left(f_{y}^{F L}-f_{y}^{F R}\right) \sin \left(\delta_{f}\right) \frac{w}{2}+\left(f_{y}^{F L}+f_{y}^{F R}\right) \cos \left(\delta_{f}\right) l_{f}-\left(f_{y}^{R L}+f_{y}^{R R}\right) l_{r} \\
&-\left(f_{x}^{F L}+f_{x}^{F R}\right) \sin \left(\delta_{f}\right) l_{f}+\left(f_{x}^{F L}-f_{x}^{F R}\right) \cos \left(\delta_{f}\right) \frac{w}{2}+\left(f_{x}^{R L}-f_{x}^{R R}\right) \frac{w}{2}
\end{aligned}
$$

where $\delta_{f}$ is the front wheel angle and $v_{x}$ is the vehicle longitudinal speed. The vehicle has a mass $m$ and yaw inertia $J_{z}$. The lateral distance from center of gravity (CoG) to front respectively rear axle are denoted $l_{f}$ and $l_{r}$. Track width is denoted $w$. The lateral tyre forces $f_{y}^{i}$ and longitudinal tyre forces $f_{x}^{i}$ with $i=\{F L, F R, R L, R R\}$ are defined according to Figure 3.

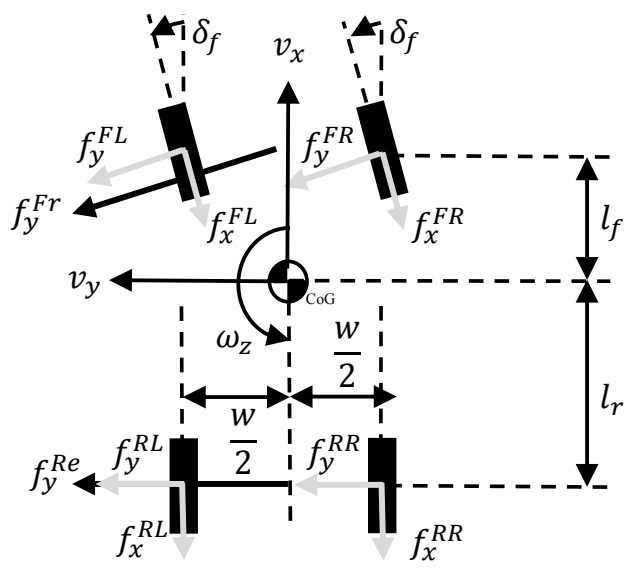

Figure 3. Vehicle model.

The Equations 1 and 2 will now be re-formulated by assuming a small front steering angle by using $\sin \left(\delta_{f}\right)=0$ and $\cos \left(\delta_{f}\right)=1$, merging lateral tyre forces to lateral axle forces, $f_{y}^{F r}$ and $f_{y}^{R e}$, and by introducing a differential brake force $F_{b}$;

$$
\begin{gathered}
m\left(\dot{v}_{y}+v_{x} \omega_{z}\right)=f_{y}^{F r}+f_{y}^{R e} \\
J_{z} \dot{\omega}_{z}=f_{y}^{F r} l_{f}-f_{y}^{R e} l_{r}+F_{b} \frac{w}{2}
\end{gathered}
$$


where the axle forces are defined

$$
f_{y}^{F r}=f_{y}^{F L}+f_{y}^{F R}, \quad f_{y}^{R e}=f_{y}^{R L}+f_{y}^{R R}
$$

and where the virtual differential brake control signal $F_{b}$ has been introduced such that

$$
F_{b}=f_{x}^{F L}+f_{x}^{R L}-f_{x}^{F R}-f_{x}^{R R}
$$

The tyre characteristics are assumed to be linear and tyre side-slip $\alpha_{f}$ and $\alpha_{r}$ to be small such that

$$
\begin{gathered}
f_{y}^{F r}=-C_{f} \alpha_{f}=-C_{f}\left(\frac{v_{y}+l_{f} \omega_{z}}{v_{x}}-\delta_{f}\right) \\
f_{y}^{R e}=-C_{r} \alpha_{r}=-C_{r}\left(\frac{v_{y}-l_{r} \omega_{z}}{v_{x}}\right),
\end{gathered}
$$

where the front and rear axle cornering stiffness $C_{f}$ and $C_{r}$ are parameters.

Further on, actuator dynamics, dead time and tyre relaxation are all lumped into first order systems with a time constant $T_{b}$ for differential braking and $T_{s}$ for steering.

$$
\dot{F}_{b}=-\frac{1}{T_{b}} F_{b}+\frac{1}{T_{b}} F_{b}^{\mathrm{req}}, \quad \dot{\delta}_{f}=-\frac{1}{T_{s}} \delta_{f}+\frac{1}{T_{s}} \delta_{f}^{\mathrm{req}}
$$

where $F_{b}^{\text {req }}$ and $\delta_{f}^{\text {req }}$ are actuator requests.

A vehicle's curvature is in general defined as

$$
\rho=\frac{\omega_{z}+\dot{\beta}}{v_{x}}, \quad \beta=\arctan \left(\frac{v_{y}}{v_{x}}\right)
$$

but for simplicity we will however here define the curvature with $\dot{\beta}$ neglected and for the calculation of curvature $\dot{\beta}$ is approximated to zero across the paper.

Selecting the state vector $x$ and input vector $u$ such that

$$
x=\left[\begin{array}{c}
v_{y} \\
\omega_{z} \\
\delta_{f} \\
F_{b}
\end{array}\right], \quad u=\left[\begin{array}{c}
\delta_{f}^{\mathrm{req}} \\
F_{b}^{\mathrm{req}}
\end{array}\right], \quad y=\rho
$$

the complete vehicle model is then expressed in state-space form as

$$
\dot{x}=A x+B u, \quad y=C x
$$




$$
\begin{gathered}
A=\left[\begin{array}{cccc}
-\frac{C_{f}+C_{r}}{m v_{x}} & \left(-v_{x}-\frac{l_{f} C_{f}-l_{r} C_{r}}{m v_{x}}\right) & \frac{C_{f}}{m} & 0 \\
-\left(\frac{l_{f} C_{f}-l_{r} C_{r}}{J_{z} v_{x}}\right) & -\left(\frac{l_{f}^{2} C_{f}+l_{r}^{2} C_{r}}{J_{z} v_{x}}\right) & \frac{l_{f} C_{f}}{J_{z}} & \frac{w}{2 J_{z}} \\
0 & 0 & -\frac{1}{T_{s}} & 0 \\
0 & 0 & -\frac{l}{T_{b}}
\end{array}\right] \\
B=\left[\begin{array}{ll}
0 & 0 \\
0 & 0 \\
\frac{1}{T_{s}} & 0 \\
0 & \frac{1}{T_{b}}
\end{array}\right], \quad C=\left[\begin{array}{lll}
0 \frac{1}{v_{x}} & 0 & 0
\end{array}\right] .
\end{gathered}
$$

The system in Equation 12 is also described in transfer function form;

$$
\begin{gathered}
y=G(s) u \\
G(s)=\left[G_{s}(s) G_{p}(s)\right]=C(s I-A)^{-1} B,
\end{gathered}
$$

where the individual transfer functions from steering and differential braking are

$$
\begin{gathered}
\rho=G_{s}(s) \delta_{f}^{\mathrm{req}}, \quad G_{s}(s)=\frac{b_{2} s^{2}+b_{1} s+b_{0}}{a_{4} s^{4}+a_{3} s^{3}+\cdots+a_{0}} \\
\rho=G_{p}(s) F_{b}^{\mathrm{req}}, \quad G_{p}(s)=\frac{c_{2} s^{2}+c_{1} s+c_{0}}{a_{4} s^{4}+a_{3} s^{3}+\cdots+a_{0}},
\end{gathered}
$$

where the polynomial coefficients are vehicle speed dependent (coefficients are listed for $70 \mathrm{~km} / \mathrm{h}$ in Table 1). At $70 \mathrm{~km} / \mathrm{h}$ the poles of the systems are positioned in $\{-6.5 \pm$ $3.2 i,-3.3,-10\}$.

\subsection{Vehicle model validation}

A validation test for the vehicle model described by Equation 12 with $F_{b}^{\text {req }}$ as input and $\omega_{z}$ as state for validation has been done at two different vehicle velocities and is shown in Figure 4 . The test was conducted by driving straight ahead at constant speed and high friction $(\mu \approx 1.0)$ and then, at $t=0 \mathrm{~s}$, requesting $F_{b}^{\text {req }}=\frac{m g}{2}$ with steering-wheel angle fixed to zero. For the real vehicle test, the steering-wheel angle, vehicle velocity, angular velocities and accelerations were all measured through the CAN bus and filtered by a $30 \mathrm{~Hz}$ low pass filter. During the tests of the real vehicle, it was observed that the ABS was partly activated indicating that friction utilization was maximized along the braked side of the car. It is also clear from the lower subplot in Figure 4 that real world vehicle exhibited a linearly decreased speed of approximately $4.5 \mathrm{~m} / \mathrm{s}^{2}$. The yawrate response 

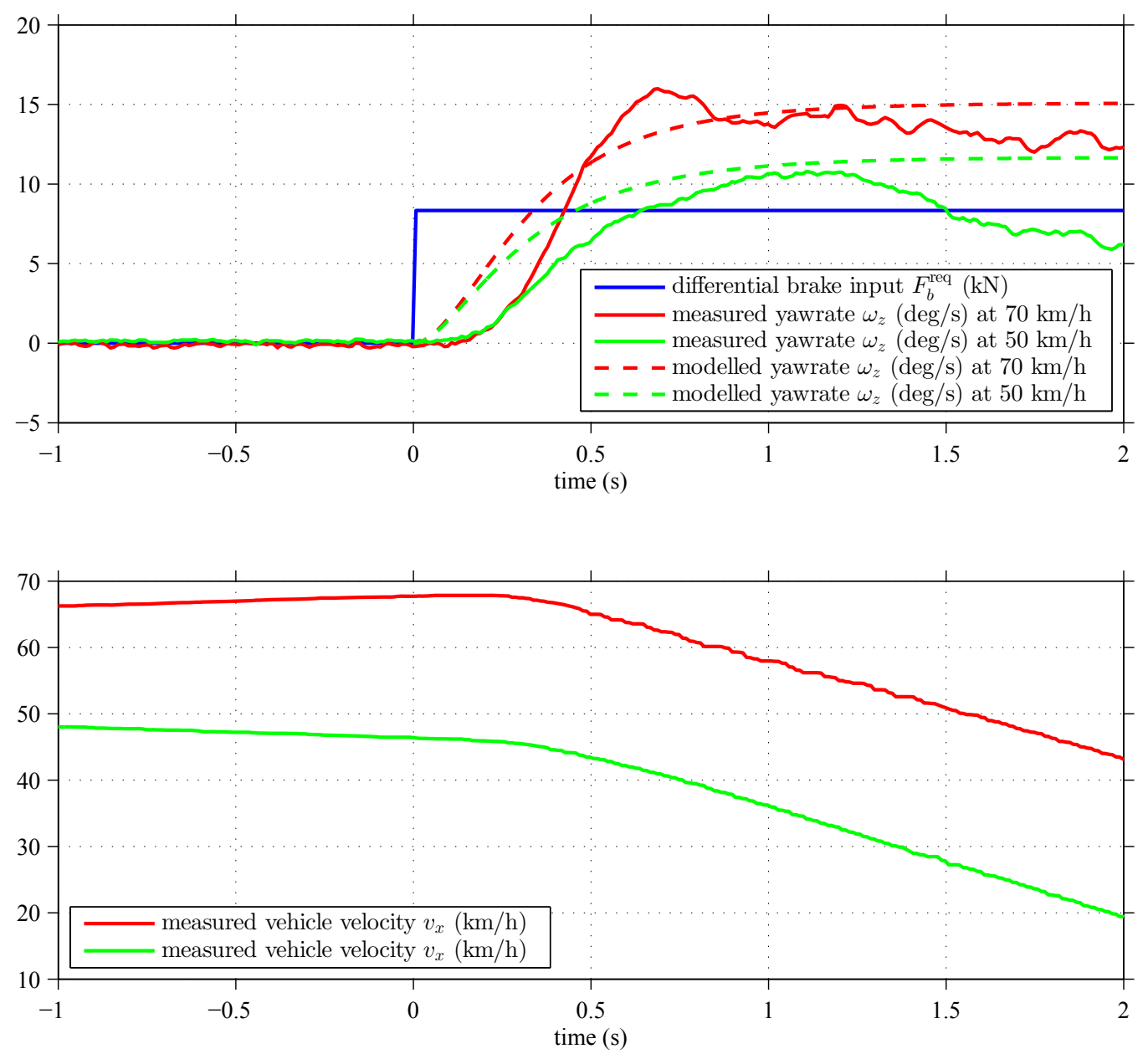

Figure 4. Step response validation test of yawrate with differential brake input at two different vehicle velocities.

from the model is however based on constant speed, which explains the deviation of yawrate at the end of the manoeuvre. $v_{x}$ is not a state in the model since we have here prioritized simplicity of a linear model. Expected model uncertainties are the axle cornering stiffnesses and the friction between brake pad and disc, and also, tyre and road. See Figure 3 for definition of physical entities and Table 1 for values of parameters.

\section{STEADY-STATE CORNERING CURVATURE}

This section is devoted to investigate and quantify the curvature that differential braking provides. By deriving the analytic expression of the filter coefficients $a 0, b 0$ and $c 0$ in Equations 17, the steady-state cornering curvature is expressed as

$$
\begin{aligned}
& \rho=G_{s}(0) \delta_{f}^{\mathrm{req}}+G_{p}(0) F_{b}^{\mathrm{req}}= \\
& \quad \frac{C_{f} C_{r} L}{C_{f} C_{r} L^{2}+m v_{x}^{2}\left(l_{r} C_{r}-l_{f} C_{f}\right)} \delta_{f}^{\mathrm{req}}+\frac{w\left(C_{f}+C_{r}\right)}{2\left(C_{f} C_{r} L^{2}+m v_{x}^{2}\left(l_{r} C_{r}-l_{f} C_{f}\right)\right)} F_{b}^{\mathrm{req}}
\end{aligned}
$$


Table 1. Vehicle parameters from [1] extended with steering parameters.

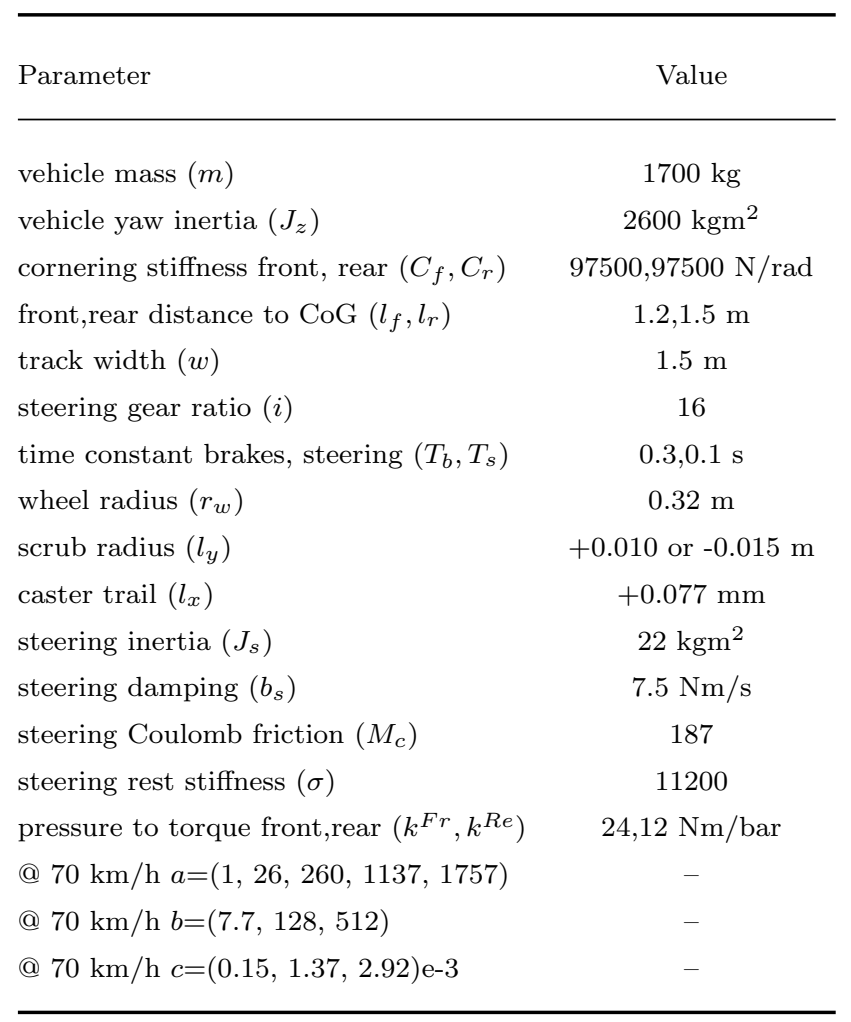

Figure 5 shows the upper bound of the linear model to what can be achieved of steadystate curvature and lateral acceleration for maximum differential brake input and steering. The maximum differential brake and steering input are assumed to be $F_{b}=\frac{m g}{2}$ and $\delta_{f}^{\text {req }}=22$ deg. When the vehicle's center of gravity height is large and track width is small, then the inner wheels decrease their vertical load significantly during lateral acceleration. The maximum differential brake force is then consequently less than $\frac{m g}{2}$. This effect is however intentionally ignored since the actual vehicle has a low height of center of gravity $(0.4 \mathrm{~m})$ and we seek expressions with as less parameters as possible. For the actual vehicle the maximum differential brake force is reduced by $13 \%$ during a steady state lateral acceleration of $5 \mathrm{~m} / \mathrm{s}^{2}$. The linear model doesn't consider friction, which for example implies that the magnitude of lateral acceleration from differential braking and steering exceeds $g \mathrm{~m} / \mathrm{s}^{2}$ for $v_{x}>30 \mathrm{~m} / \mathrm{s}$ and $v_{x}>8 \mathrm{~m} / \mathrm{s}$ respectively. Since the vehicle is understeered $\left(l_{r} C_{r} \geq l_{f} C_{f}\right)$, the largest curvature during zero steering angle is found

$$
\rho_{\max }=\lim _{v_{x} \rightarrow 0} \rho=\frac{w\left(C_{f}+C_{r}\right)}{2\left(C_{f} C_{r} L^{2}\right)} F_{b} \leq \frac{w\left(C_{f}+C_{r}\right) \mu m g}{4\left(C_{f} C_{r} L^{2}\right)}
$$

In Equation 20 it is assumed that the largest differential brake force is $F_{b}=\frac{\mu m g}{2}$, which is a conservative estimate due to the intentionally ignored effect of load transfer. From Equation 20 it follows that the maximum curvature is limited and proportional to friction. For the vehicle in this study the upper bound of cornering curvature during high friction $(\mu=1)$ is $0.0171 / \mathrm{m}$ which corresponds to a cornering radius of $59 \mathrm{~m}$. Keeping the assumption of $\mu=1$, Figure 5 gives us that the curvature is bounded between $0.0111 / \mathrm{m}$ and $0.0171 / \mathrm{m}$. The maximum curvature obtained by differential braking is smaller than 

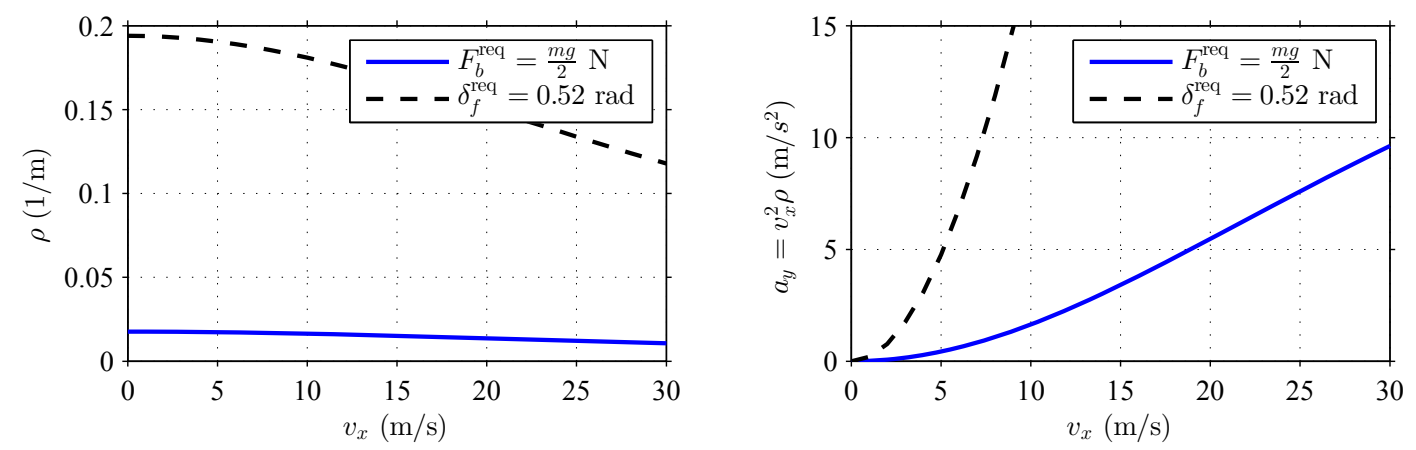

Figure 5. Linear model according to Equation 19 of steady-state curvature and lateral acceleration for either maximum differential brake or maximum steering input.

for steering. For vehicle speeds below $50 \mathrm{~km} / \mathrm{h}$ the lateral acceleration will not exceed 3 $\mathrm{m} / \mathrm{s}^{2}$ when using differential braking. The corresponding speed for steering is $16 \mathrm{~km} / \mathrm{h}$. If "normal driving" is considered as being able to reach lateral acceleration magnitudes of 3 $\mathrm{m} / \mathrm{s}^{2}$, differential braking cannot meet that. The reduced cornering ability for differential braking will in turn increase the need for a careful path and speed planning.

\section{OBSERVATIONS OF SCRUB RADIUS EFFECT}

\subsection{Hands-on versus hands-off the steering wheel}

The curvature retrieved from differential braking in previous sections assumes zero steering angle. However, a brake force on a front wheel will, together with a moment arm mentioned to as scrub radius, induce an alignment wheel torque, which will influence steering and in turn the cornering curvature. This section seeks to experimentally quantify the scrub radius effects of the resulting curvature.

The scrub radius is a lateral displacement between the tyre's center of rotation, caused by the kingpin axis intersection with with the road plane, and the centerline of the tyre. When the center of rotation is outside of the centerline of the tyre, the scrub radius is defined as negative, otherwise it is positive. See Figure 6 for clarification. The differential brake test described in Section 3.2 was conducted with hands-on the steering-wheel, where the driver fixed the steering-wheel angle to zero. The test is here repeated but now with hands-off the steering-wheel i.e. no steering-wheel torque is applied during the test event. As shown in Figure 7, the steering-wheel angle is no longer zero caused by the (positive) scrub radius. At the end of the manoeuvre, when vehicle speed is lower, the steering angle is significantly increased up to 65 degrees. This will in turn, contribute to even higher cornering curvature. The large steering-wheel angle corresponds to approximately 4 degrees wheel angle, which confirms that the small angle approximation made in Equations 1 and 2 is relevant. The experiment clearly demonstrate that the scrub radius effect substantially influences the physical limit of maximum cornering curvature. It should be mentioned that the scrub radius effect can be modeled and included in the vehicle model. This would be beneficial since such model predicts the curvature better. The scrub radius is however uncertain since it for example varies for different wheel hubs, and consequently, the model becomes sensitive for the scrub radius parameter. 

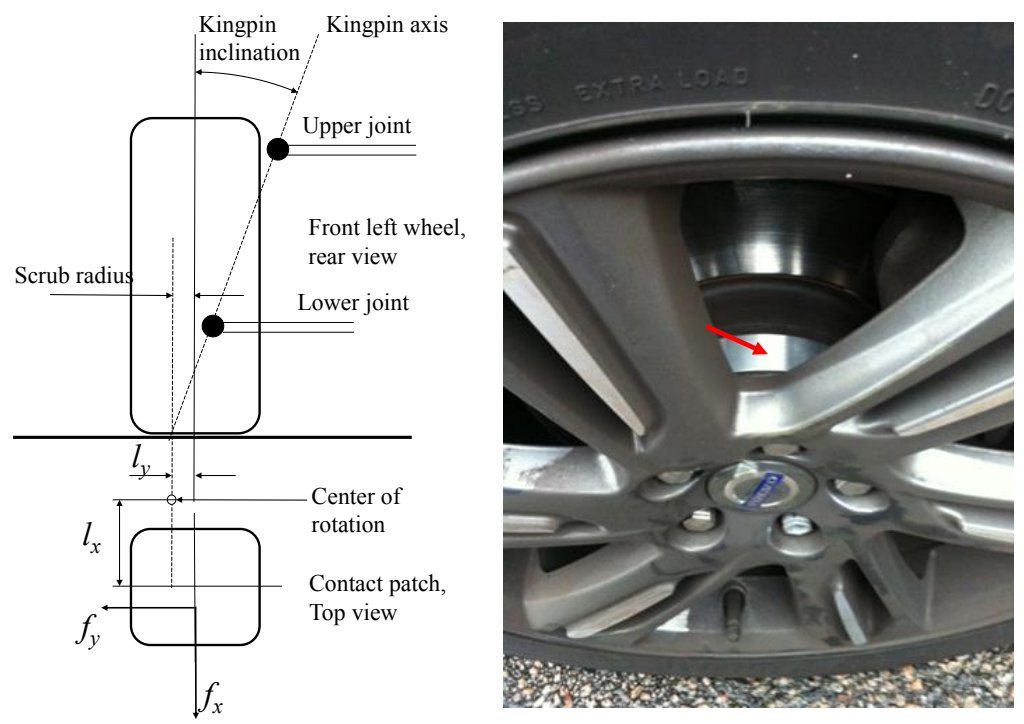

Figure 6. The scrub radius and here illustrated as negative (left). Spacers used to experimentally vary the scrub radius (right).
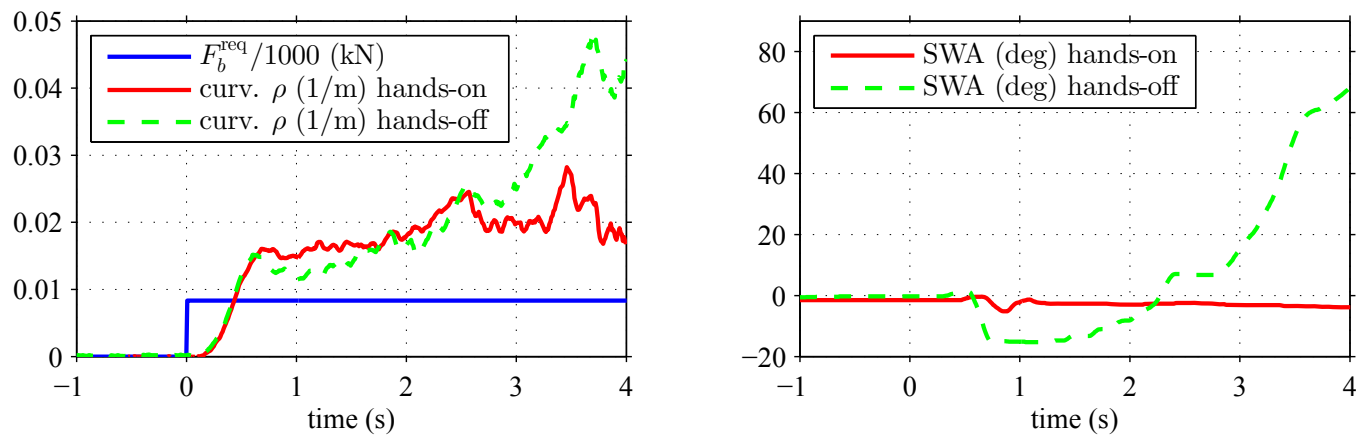

Figure 7. Step response test of curvature (left) and steering-wheel angle (right) for zero steering-wheel angle (hands-on) and zero steering-wheel torque (hands-off).

\subsection{Perturbation of the scrub radius}

The observation done in Section 5.1 showed that the scrub radius, when releasing the steering-wheel, increased the steering alignment torque and the cornering curvature significantly. The scrub radius was $+10 \mathrm{~mm}$. To further test the cornering ability for various scrub radius, it was changed to $-15 \mathrm{~mm}$. The modification of the vehicle has been made possible due to the use of so called spacers, which is a device mounted inside the rim which moves the contact patch outwards and hence creating the positive scrub radius. The hands-off differential brake test done in Section 5.1 was repeated with the two different scrub radii. As seen in Figure 8, the negative scrub radius will generate negative steering angles, which will counteract the cornering curvature from yaw torque, ending up in a smaller magnitude of total cornering curvature. The perturbation test demonstrates the high sensitivity of the cornering curvature with respect to different scrub radius. 
Figure 8 demonstrates that the scrub radius in particular influences the curvature for low vehicle speed.
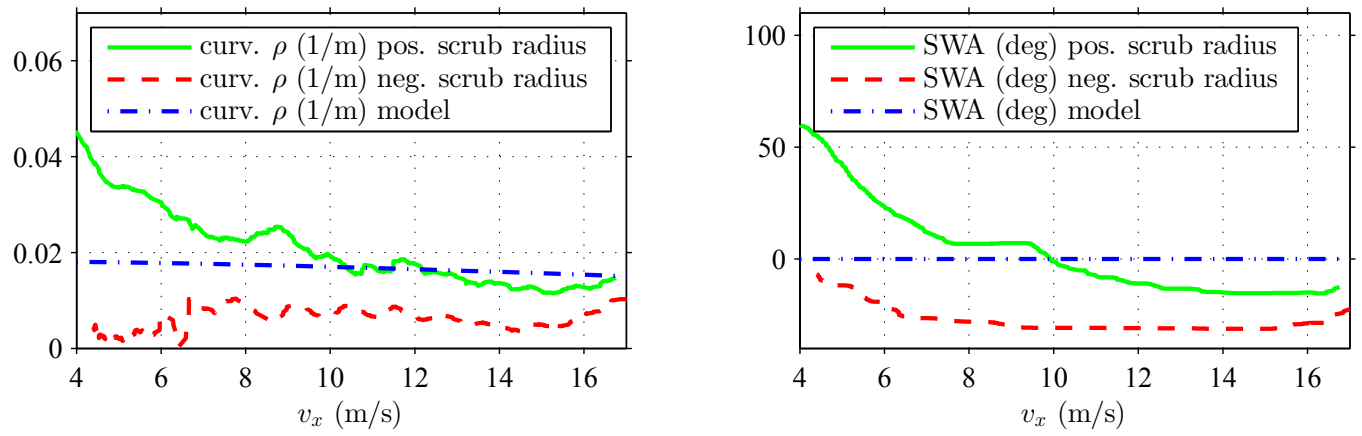

Figure 8. Observations of curvature and steering-wheel angle for positive versus negative scrub radius for $F_{b}^{\text {req }}=$ $\frac{m g}{2}$. The blue dashed line correspond the steady-state model described in Equation 19.

\section{VEHICLE MODEL WITH SCRUB-RADIUS}

\subsection{Vehicle model derivation}

In order to get a conceptual understanding of the scrub radius effect observed in the previous section, e.g understand how curvature and steering angle depend on the wheel suspension, this section models the vehicle including the steering system. Of special interest is the curvature capability caused by the wheel suspension design. The steering system, which can be described by a second order differential equation [5] has been provided with front tyre longitudinal force input such that

$$
J_{s} \ddot{\delta}_{f}+b_{s} \dot{\delta}_{f}+l_{x} f_{y}^{F r}+M_{f}=l_{y}\left(f_{x}^{F L}-f_{x}^{F R}\right) .
$$

When there is an asymmetry in the two front tyre longitudinal forces, an alignment torque is generated due to the scrub radius moment arm $l_{y}$. In addition to inertia, $J_{s}$, and damping, $b_{s}$, of the steering system, the torque equilibrium contains alignment torque due to lateral force and a moment arm $l_{x}$. This arm is the sum of the caster trail and the pneumatic trail, i.e. the distance along the tyre centerline from the wheel's center of rotation at the road to the point where the lateral force acts. This caster trail depends on the actual wheel suspension design and the pneumatic trail of the tyre. The moment arms $l_{x}$ and $l_{y}$ are assumed to be constants and are both illustrated in Figure 6 . In Equation 21, the lift effect [6], i.e. the influence from the normal load of the steering angle has been neglected since it during the work was found to be low. The friction torque $M_{f}$ in the entire steering system has been modeled by the Dahl friction model [7] such that

$$
\dot{M}_{f}=\sigma \operatorname{sign}\left(1-\frac{M_{f}}{M_{c}} \operatorname{sign}\left(\dot{\delta}_{f}\right)\right) \dot{\delta}_{f}
$$

where $M_{c}$ is the Coulomb friction torque and $\sigma$ is the rest stiffness. In Figure 9, the steering friction torque is shown for the signal $\delta_{f}(t)=0.1 \sin (2 \pi t) \mathrm{rad}$. 


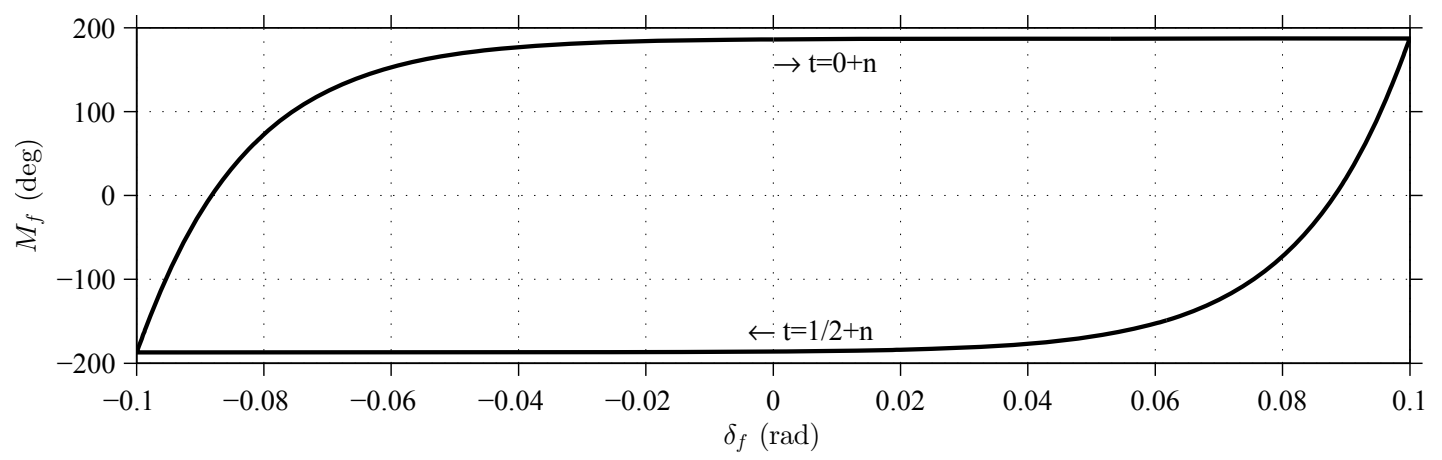

Figure 9. The hysteresis map from the Dahl friction model.

From Equation 21 it is obvious that front wheel braking can be used to control the steering angle. Apart from controlling the steering angle, an asymmetry in front and rear brake forces generates a yaw torque. We will now reformulate the vehicle model to also include the effect that the wheel steering angle changes during differential braking.

To capture the difference of braking the front and rear wheels, the lumped brake force $F_{b}$ cannot be used any longer. Ideally, all four individual brake forces should be used, but to keep complexity lower, the braking is here assumed to be done only at one side of the vehicle. To simplify the notation, the left side is selected for the derivation. The brake actuator first order system dynamics is here neglected to keep complexity low. Selecting the state vector $x_{s}$ and input vector $u_{s}$ such that

$$
x_{s}=\left[\begin{array}{c}
v_{y} \\
\omega_{z} \\
\delta_{f} \\
\dot{\delta}_{f}
\end{array}\right], \quad u_{s}=\left[\begin{array}{c}
f_{x}^{F L} \\
f_{x}^{R L} \\
M_{f}
\end{array}\right], \quad y_{s}=\left[\begin{array}{c}
\rho \\
\delta_{f}
\end{array}\right]
$$

The complete vehicle model is then expressed in state-space form as

$$
\begin{gathered}
\dot{x_{s}}=A_{s} x_{s}+B_{s} u_{s}, \quad y_{s}=C_{s} x_{s} . \\
A_{s}=\left[\begin{array}{cccc}
-\frac{C_{f}+C_{r}}{m v_{x}} & \left(-v_{x}-\frac{l_{f} C_{f}-l_{r} C_{r}}{m v_{x}}\right) & \frac{C_{f}}{m} & 0 \\
-\left(\frac{l_{f} C_{f}-l_{r} C_{r}}{J_{z} v_{x}}\right) & -\left(\frac{l_{f}^{2} C_{f}+l_{r}^{2} C_{r}}{J_{z} v_{x}}\right) & \frac{l_{f} C_{f}}{J_{z}} & 0 \\
0 & 0 & 0 & 1 \\
0 & \frac{l_{x} l_{f} C_{f}}{J_{s} v_{x}} & -\frac{l_{x} C_{f}}{J_{s}}-\frac{b_{s}}{J_{s}}
\end{array}\right]
\end{gathered}
$$




$$
B_{s}=\left[\begin{array}{ccc}
0 & 0 & 0 \\
\left(\frac{w}{2 J_{z}}+\frac{l_{y} l_{f}}{l_{x} J_{z}}\right) & \frac{w}{2 J_{z}} & 0 \\
0 & 0 & 0 \\
\frac{l_{y}}{J_{s}} & 0 & \frac{1}{J_{s}}
\end{array}\right], \quad C_{s}=\left[\begin{array}{cccc}
0 & \frac{1}{v_{x}} & 0 & 0 \\
0 & 0 & 1 & 0
\end{array}\right]
$$

When braking at the right side of the vehicle, the sign of $B_{s}$ should be changed. Note that the friction torque, due to its nonlinear nature, is an input to the linear system.

\subsection{Model validation}

Figure 10 shows the steady-state solution of system 24, which now includes friction torque, caster trail and scrub radius, together with the measured data. As seen from the figure, the model outputs resemble the results from the measurements, i.e. the steering angle and curvature for various vehicle speed. It is seen from both the model output and measurement that the steady-state steering angle is approximately doubled when decreasing vehicle speed from $15 \mathrm{~m} / \mathrm{s}$ to $10 \mathrm{~m} / \mathrm{s}$. It was however observed during the work that the modeling of the friction was important to get a good match with the measured data. Caster trail and scrub radius are parameters that can be retrieved relatively easy from e.g Adams modeling tools, drawings etc. Friction torque in the steering is significantly harder to predict. The parameters of the friction model was identified to get as good match as possible, but Figure 10 shows also the result from a perturbed friction with a $50 \%$ increase of the Coulomb friction torque. High Coulomb friction torque when there is a positive scrub radius results in smaller magnitude of curvature since the friction resists the front wheels to be steered in the intended direction. On the contrary, high Coulomb friction torque when there is a negative scrub radius results in larger magnitude of curvature. From the figure it is evident that a correct modeled steering friction is important in order to get a valid vehicle model. Noteworthy, it was subjectively observed during the measurements that just tiny friction between driver's hand and the steering-wheel did reduce or stop the rotation of the steering-wheel.
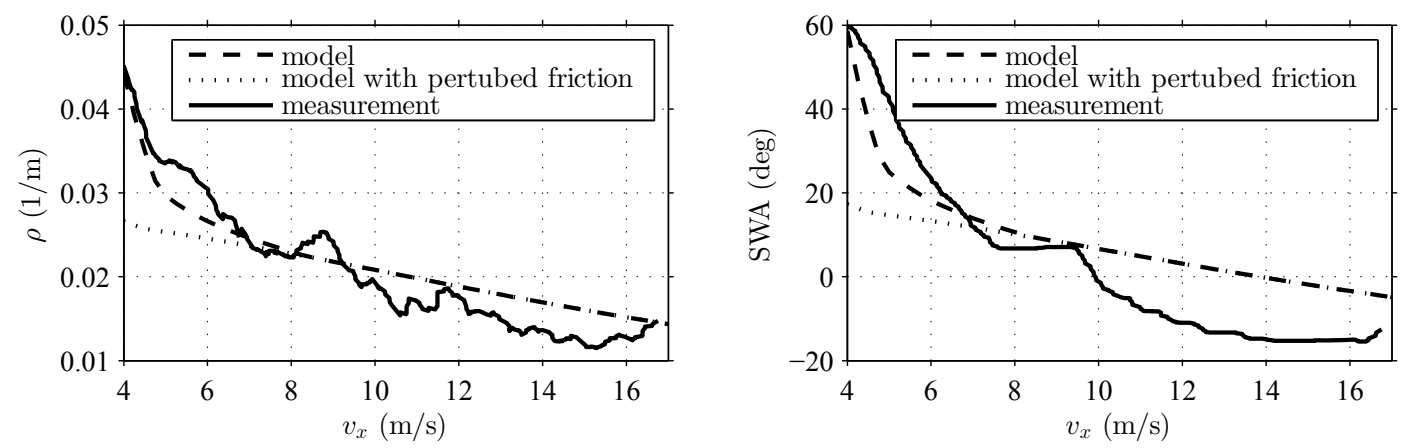

Figure 10. Steady-state curvature and steering-wheel angle from system 24 and measurement for $+10 \mathrm{~mm}$ scrub radius for $f_{x}^{F L}=f_{x}^{R L}=\frac{m g}{4}$. The model is simulated with two different parameterizations of steering friction. 


\subsection{Maximum curvature capability}

The non-linearity of friction in the steering model make it more difficult to express steady state solutions. For example; the steady state solution of the steering angle depends on previous states, which complicates the interpretation and assessments of the steady state solution. Further on in this section, we will neglect the steering friction to be able to derive simple analytical expressions. The analytical expressions will be used to understand, at a conceptual level, the physics that determines the cornering during differential braking. The steady-state curvature from Equation 24 when $M_{f}=0$ is derived

$$
\rho=\frac{4 l_{y} l_{f}+2 l_{y} l_{r}+l_{x} w}{2 l_{x} l_{r} m v_{x}^{2}} f_{x}^{F L}+\frac{w}{2 l_{r} m v_{x}^{2}} f_{x}^{R L} .
$$

Note that the models steady-state curvature does not depend on the axle's cornering stiffness. The lateral velocity is however dependent on cornering stiffness (not shown in this paper).

The expression for the steady-state steering angle $\delta(\rho)$ is long and not suitable to be presented, but it can through Equation 21 and 7 be expressed as $\delta\left(v_{y}, \omega\right)$ instead

$$
\delta_{f}=\frac{l_{y}}{C_{f} l_{x}} f_{x}^{R L}+\frac{v_{y}+l_{f} \omega_{z}}{v x} .
$$

Note that if the FL wheel is not braked, the FL wheel will be pointed in the front axle slip direction.

As seen from Equation 27 the curvature will be increased for the front (left) wheel braking, if the vehicle's design parameters is selected such that $\left(4 l_{y} l_{f}+2 l_{y} l_{r}+l_{x} w\right)>0$ . Otherwise the curvature will decreased. If the curvature is to be maximized, $\left(4 l_{y} l_{f}+\right.$ $\left.2 l_{y} l_{r}+l_{x} w\right)$ must be greater than zero together with maximum brake forces on the (left) side. Assuming that maximum brake forces are applied such that $f_{x}^{F L}=f_{x}^{R L}=\frac{m g}{4}$ the maximum curvature is expressed as

$$
\rho_{\max }=\frac{\mu g\left(l_{y}\left(2 l_{f}+l_{r}\right)+l_{x} w\right)}{4 l_{x} l_{r} v_{x}^{2}} .
$$

\subsection{System design constraints}

The ratio between scrub radius and the caster trail is now defined as

$$
\xi=\frac{l_{y}}{l_{x}} .
$$

From Equation 29, together with the fact that the steady-state lateral acceleration is quadratic proportional to vehicle speed, it is clear that the steady-state lateral acceleration capability is independent from vehicle speed such that

$$
a_{y, \max }=\frac{\mu g\left(\xi\left(2 l_{f}+l_{r}\right)+w\right)}{4 l_{r}} .
$$

Hence, it can be concluded that the following items contribute to a large lateral acceleration capability: 
(1) Large vehicle width

(2) Center of gravity close to the rear axle

(3) Large positive scrub radius

(4) Small positive caster trail

Note that when caster trail approaches $0^{+}$, the linear vehicle model gives infinitely high lateral acceleration capability and steering angle, which of course is not possible since tyre forces in practice are limited.

When designing the self-driving vehicle Equation 31 can be used to conceptually find vehicle parameters to get a desired lateral acceleration capability. If the scrub radius should be designed in order to reach a lateral acceleration capability of $a_{y, \max }=3 \mathrm{~m} / \mathrm{s}^{2}$ then a vehicle with parameters according to Table 1 would require $\xi>=0.09$ and in turn a positive scrub radius $l_{y}>=+7 \mathrm{~mm}$.

Looking at the poles in Figure 11, the vehicle is stable for the positive scrub radius tested $(+10 \mathrm{~mm})$. However when the caster trail switches sign from positive to negative, the system becomes unstable due to poles in the right half complex plane. Since unstable system is more difficult to control, negative caster trail is normally avoided.

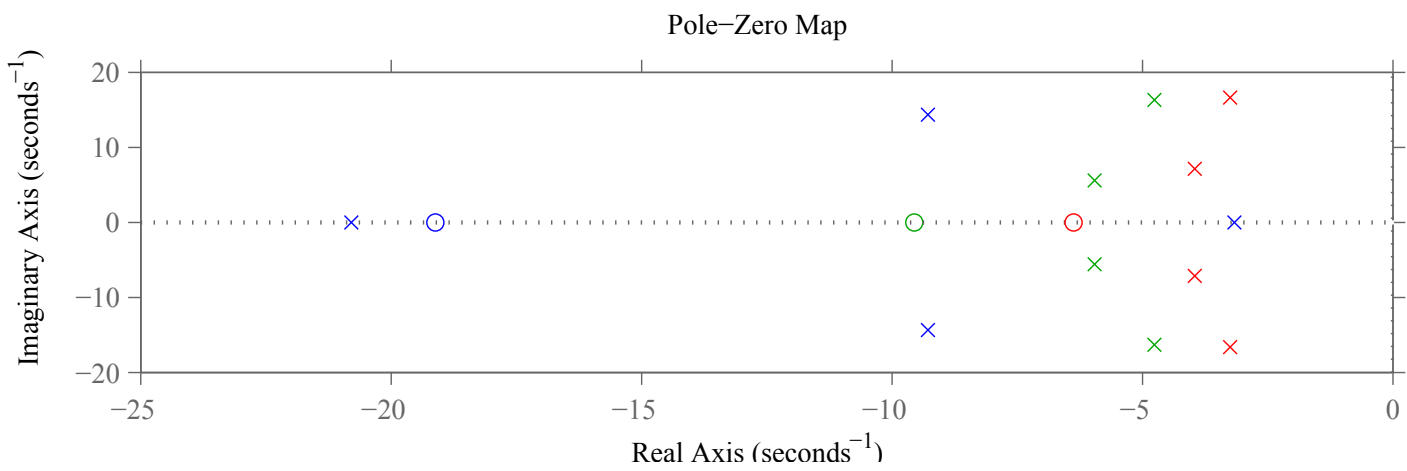

Figure 11. Poles and zeros from system 24 for $+10 \mathrm{~mm}$ scrub radius and $+77 \mathrm{~mm}$ caster trail at $6 \mathrm{~m} / \mathrm{s}$ (blue), $12 \mathrm{~m} / \mathrm{s}$ (green), $18 \mathrm{~m} / \mathrm{s}$ (red).

\section{CURVATURE CONTROLLER DESIGN}

In order to tackle the steering fault described in the Introduction, we will here design an on-board curvature controller to be used after the occurrence of a fault when the vehicle is driven in autonomous mode. Note again that during the control event, the steering actuator nor the driver are assumed to steer the vehicle. When the fault is detected, the curvature controller starts to request differential brake force.

\subsection{Selection of control design}

In this work, two vehicle models have been developed. This first one (Equation 12) has no model of the wheel suspension mechanism that has shown to be important during control of curvature and hands-off the steering wheel. The model contains cornering stiffness parameters which are uncertain. The second model (Equation 24) has the suspension mechanism modeled, but suffers from that the steering system is hard to model due to steering friction uncertainty. Variation in steering friction will make it hard to 
design robust controller, therefor the control design will be based on the first model (Equation 12).

\subsection{Control design}

The initial steering angle and its evolution, e.g. depending on the uncertain scrub radius, is here considered as a disturbance. By rearranging Equation 19, the differential brake request for static conditions is formulated as

$$
F_{b}^{\mathrm{req}}=\frac{1}{G_{p}(0)} \rho_{\mathrm{req}}-\frac{G_{s}(0)}{G_{p}(0)} \delta_{f},
$$

where the first term in Equation 32 will be the feedforward compensator for the requested curvature $\rho_{\text {req }}$ and the second term the feedforward compensator for rejecting the disturbance $\delta_{f}$. The rejection of the steering disturbance is possible since $\delta_{f}$ is measured (through steering-wheel angle sensor) and the influence of the curvature is modeled.

Due to model uncertainties and neglected dynamics for both $G_{p}(0)$ and $G_{s}(0)$, there will be a control error and disturbance will not be completely eliminated. Therefore a feedback loop is needed along with the two compensators. See [8] where the feedforward and feedback control design are separated. Equation 32 is now expressed

$$
\begin{gathered}
F_{b}^{\mathrm{req}}(s)=\frac{2\left(C_{f} C_{r} L^{2}+m v_{x}^{2}\left(l_{r} C_{r}-l_{f} C_{f}\right)\right)}{w\left(C_{f}+C_{r}\right)} \rho_{\text {req }}-\frac{2 C_{f} C_{r} L}{w\left(C_{f}+C_{r}\right)} \delta_{f}+C(s) e \\
C(s)=K_{p}\left(1+\frac{1}{s T_{i}}+\frac{s T_{d}}{1+s T_{d} / N}\right) \\
e=\rho_{\text {req }}-\rho=\rho_{\text {req }}-\frac{\omega_{z}}{v_{x}} .
\end{gathered}
$$

To limit the high frequency gain of the derivative term, a low pass filter has been incorporated in the design of $C(s)$ which gives an upper bound of $K_{p} N$. Finally, the unfiltered curvature request is passed through a set-point rate-limiter filter, which protects for undesired derivative kicks during abrupt step changes in the curvature requests. See [10] for PID filter design to avoid over shoots. The control structure in its entirety is illustrated in Figure 12. The control $F_{b}$ is virtual and needs to be allocated to wheel individual brake pressures. The sign of $F_{b}^{\text {req }}$ determines which side should be actuated. There is a freedom in how $F_{b}^{\mathrm{req}}$ is distributed among the two tyres. The distribution is here done to preserve equal friction utilization and hence provide equal lateral force margins for good robustness. The lateral force component is here for simplicity neglected.

Neglecting the wheel dynamics, the brake pressure requests $p^{\text {req }}=$ $\left[p^{F L \text {,req }} p^{F R \text {,req }} p^{R L \text {,req }} p^{R R \text {,req }]^{T} \text { to wheels are finally defined as }}\right.$

$$
p^{\mathrm{req}}=\left[\begin{array}{llll}
0 & \frac{l_{r} r_{w}}{L k^{F r}} & 0 & \frac{l_{f} r_{w}}{L k^{R e}}
\end{array}\right]^{T} F_{b}^{\mathrm{req}} \quad \text { if } F_{b}^{\mathrm{req}} \leq 0
$$




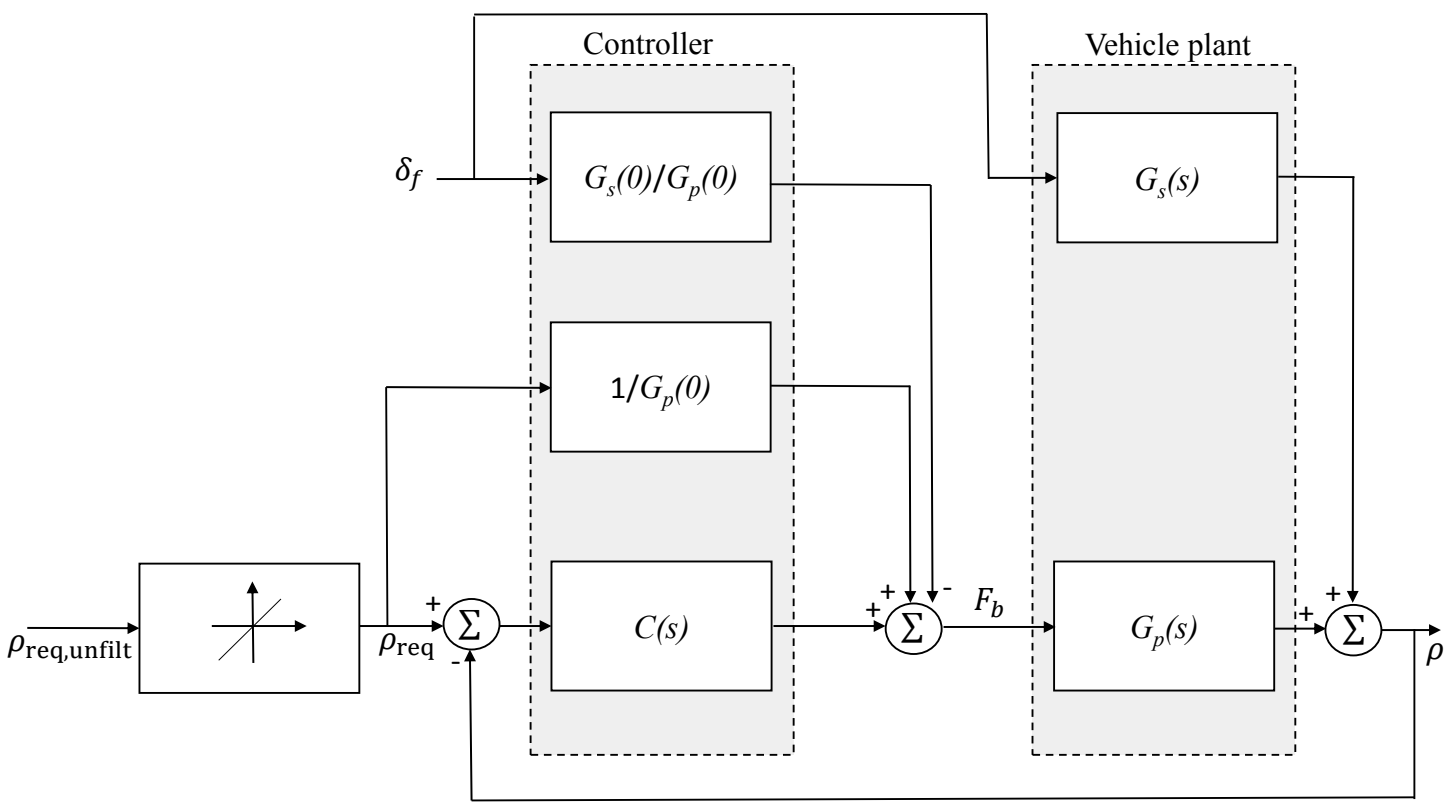

Figure 12. Curvature control structure consisting of two feedforward compensators, one feedback controller and a set-point filter.

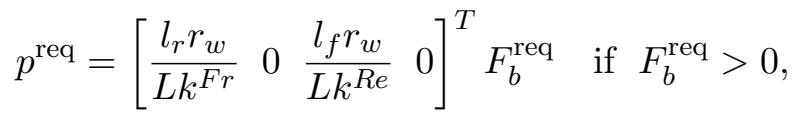

where $r_{w}$ is the wheel radius and $k^{F r}$ and $k^{R e}$ are conversion factors from brake pressures to wheel torques depending on wheel geometry and hydraulic brake cylinders. The brake pressure request is send to a brake control unit, which is provided with a slip controller to prevent from wheel lock and limit longitudinal slip to approximately $10 \%$.

The tuning of the PID controller was done with the Ziegler-Nichols method [11] where frequency information of the controlled system was used to determine the controller coefficients.

\subsection{Test of the control design}

To test the controller, we will now revisit the special case discussed in Section 2 and shown in Figure 1. The vehicle is driving straight ahead at $70 \mathrm{~km} / \mathrm{h}$ and a steering failure occurs before a 200 meter road radius. When the curve is entered, a constant curvature request, corresponding to the actual road curvature ahead, is sent to the curvature controller. The curvature request is not updated during the control, which is a simplification in the test set up. The step response with the developed controller is shown in Figure 13. The time to reach $63 \%$ of the final curvature is by the closed loop controller reduced from approximately 0.4 to $0.3 \mathrm{~s}$ compared with the open loop response, but at the expense of an overshoot. The level of overshooting is a tuning issue, but here fast response has been prioritized to compensate for delays. The control error converge to zero and the remaining noise is caused by the wheel slip controller which work close to the slip limit.

The resulting path is shown in Figure 14. The vehicle stays within the pre-defined one meter lateral road margin. Due to the response time of the differential braking, the path is shifted to the right in the figure and a constant tracking error remains during 


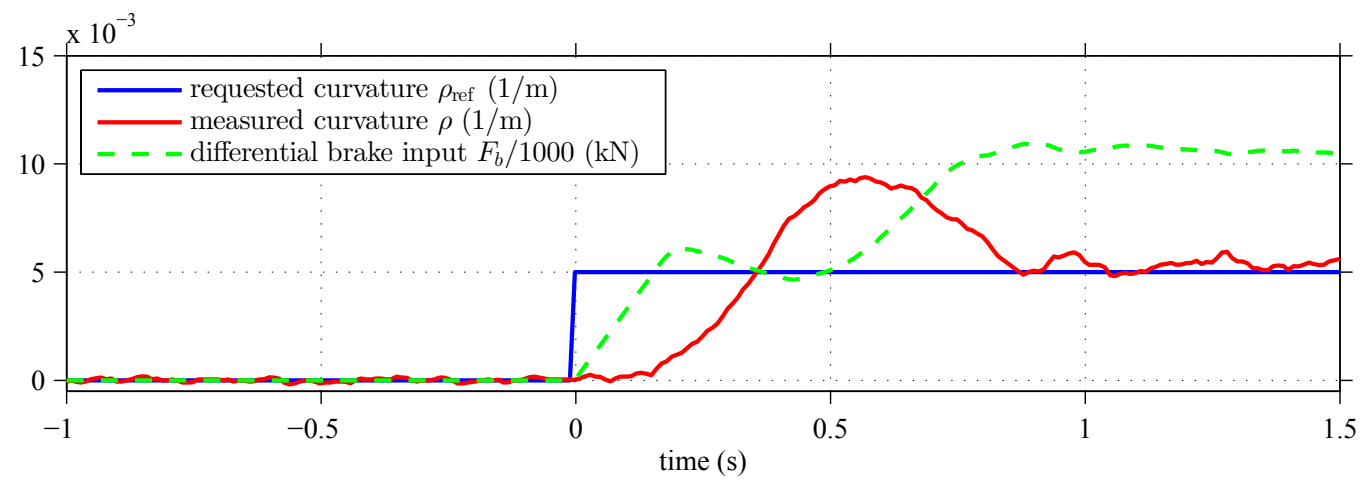

Figure 13. Close loop control of curvature starting at $t=0 \mathrm{~s}$.

the control event. This strengthen the arguments that the curvature request should be updated during the control, i.e. close the loop upon position and orientation relative to the road, if that is possible.

Differential braking could lead to instability when braking hard on a rear wheel, which is not investigated in this paper. It is assumed that there exist a stability system monitoring and acting when margins to instability is below a threshold.

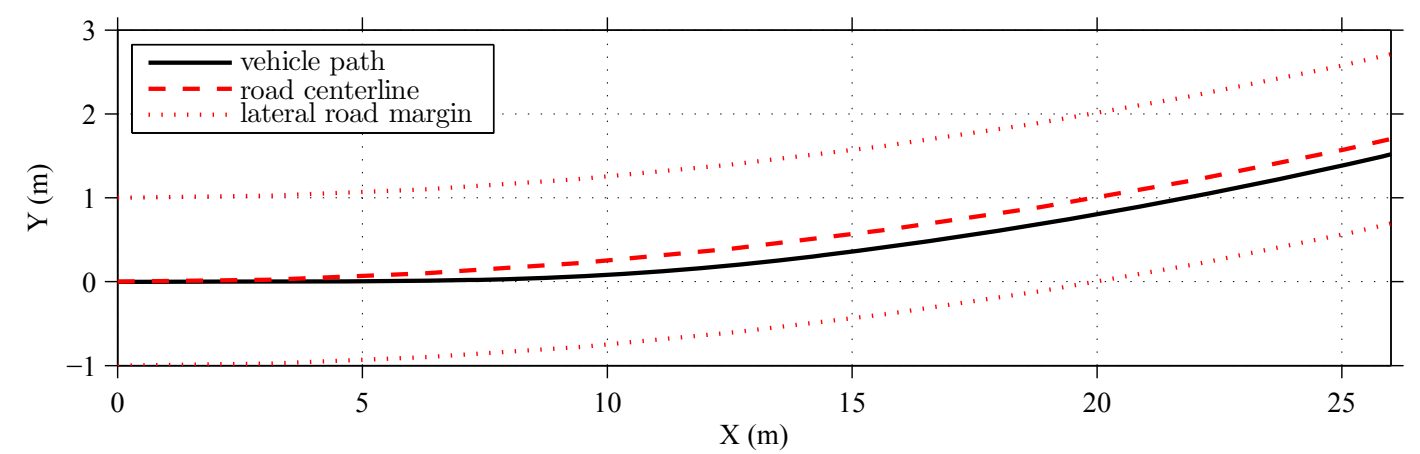

Figure 14. Resulting path from the closed loop control of curvature starting at $X=0 \mathrm{~m}$.

\section{CONCLUSIONS}

Analysis of the developed vehicle models and tests in real world vehicle has shown that differential braking could be used as an alternative to front axle steering for fault-tolerant control of self-driving vehicles. There are however physical limits on how large curvature and lateral acceleration could be achieved compared with steering. Compared with steering, differential braking can in general not provide as much lateral acceleration for lower speeds. Large curvature needs large longitudinal differential brake forces, which makes the curvature directly road friction dependent. Having said that, differential braked vehicles must plan and adapt their speed to not exhibit too large lateral accelerations. There are two cases of differential braking; either hands-off (torque free) the steering wheel or hands-on (torque from human driver or steering actuator is engaged). The hands-off case results in a curvature which is sensitive to wheel suspension parameters since an unsymmetrical front wheel braking induces a change in front steering angle. 
For the hands-off case, the scrub radius is an important wheel suspension parameter. Negative scrub radius gives unacceptable cornering capabilities, while a relative large positive scrub radius is an appropriate design alterative for acceptable cornering capabilities. The paper has shown how the scrub radius should be designed to meet curvature requirements. Apart from the scrub radius, the friction in the steering system has a key role for the curvature capability of the vehicle. Large friction will reduce the steering angle. Large friction together with positive scrub radius will also reduce the curvature capability.

When selecting the control method it is crucial to get insight about which parameters are uncertain. Due to the uncertainty in the steering system model, and in particular the steering friction, the selected control concept do not rely on the steering system.

Future work is suggested to be devoted on the evaluation of the many different combinations of manoeuvres that may occur, e.g. the aggressive special case that was introduced in Section 2.

\section{References}

[1] Jonasson M, Thor M. Steering redundancy for self-driving vehicles using differential braking. Proceedings of the 13th International Symposium on Advanced Vehicle Control; 2016. Munich, Germany.

[2] Pilutti P, Ulsoy G, Hrovat D. Vehicle steering intervention through differential braking. Journal of Dynamic Systems, Measurement, and Control; 1998. Vol. 120(3). p. 314(8).

[3] Moshchuk NK. et al. Collision avoidance maneuver through differential braking. Patent US 20130030651 A1; 2013.

[4] Rajamani R. Vehicle Dynamics and Control. Springer US; 2012.

[5] Yih P, Ryu J, Gerdes JC. Vehicle state estimation using steering torque. American Control Conference; 2004. Vol. 3. p. 2116-2121.

[6] Katzourakis DI. Driver steering support interfaces near the vehicle's handling limits. PhD Thesis. Netherlands: TU Delft; 2012.

[7] Drincic B. Mechanical models of friction that exhibit hysteresis, stick-slip, and the stribeck effect. PhD Thesis. The US: The University of Michigan; 2012.

[8] Brosilow C, Joseph B. Techniques of model based control. Prentice Hall PTR; 2002.

[9] MacAdam C, Ervin, R. Differential braking for limited-authority lateral maneuvering. IDEA Program Final Report, University of Michigan Transportation Research Institute; 1998.

[10] Hägglund, T. Signal filtering in PID control.IFAC Conference on Advances in PID Control; 2012. Brescia, Italy.

[11] Ziegler J, Nichols N. Optimum settings for automatic controllers. Transactions of the A.S.M.E.; 1942. Vol. 5(11). p. 759768. 